\title{
Hakka Traditional Daily Utensils under the Perspective of Eco-design
}

\author{
Jiali Yuan \\ Sanming University \\ Sanming, China 365004
}

\author{
Yuzhou Wu \\ Huaiyin Institute of Technology \\ Huai' an, China 223001
}

\begin{abstract}
This paper aims to explore the ecological design thought contained in Hakka traditional daily utensils, taking systematic theory as the basis to conduct system analysis of utensils" "material selection - production - use cycle" and design theories. It comes to the following conclusion: first, the Hakka traditional utensils are made from local materials with the characteristics of ecological protection; second, in the production process, the production links are compressed to reduce the use of materials, which has reduced the impact on environment; third, in the course of use, the Hakka people are good at repair and reuse, thus the utensils' service life are extended; fourth, with respect to the design theories, plain articles are produced for practical use to save resources and manpower. Through the systematic research, it can be concluded that the Hakka traditional utensils have rich ecological design thought, which have great enlightenment to contemporary design.
\end{abstract}

Keywords-Hakka traditional daily utensils; eco-design; low energy consumption; life cycle; produce articles for practical use

\section{INTRODUCTION}

Hakka traditional utensils carry with Hakka people's cultural characteristics of life in the simple form, practical function, local materials, and reasonable skills. From the remained Hakka traditional daily utensils, they contain a wealth of eco-design ideas, which not only reflect in the localized material selection with low environmental impact, low-energy consumption production techniques and the use mode which enhances efficiency, but also reflect in the simple, plain, and economical design theories which produced articles for practical use.

\section{SELECTION OF MATERIAL WITH LOW ENVIRONMENTAL IMPACT}

In the process of design, the impact on environment can be reduced through good design, thus the choice of materials is extremely critical. The selection method of eco-friendly material is "starting from the environment, the natural and appropriate local materials should be used as long as possible." [1] There will be multiple advantages to select the abundant natural materials for design: first, the abundant materials are mostly adapt to local climatic conditions, which are renewable and easy to be regenerated and supplemented, having little impact on the entire ecosystem; second, it is easy to obtain the regional materials, reducing transport, loss and other additional

Fund Project: Achievement of Fujian Province Social Science Planning Project (FJ2015C100); Achievement of Fujian Province Young and Middleage Teachers Education Research Project(JAS160496).. energy consumption, and the cost is reduced as well; third, the material itself is adapt to climatic conditions, and its functional performance will not be lowered due to environment.

Most of the materials of Hakka traditional daily utensils are selected from the local place, such as clay and stone that are easy to get, and bamboo, wood, rattan and other materials that are easy to grow "Fig. 1": Hakka people have shaped a variety of practical utensils by virtue of their skills and clever thinking, making applications depending on the materials, and adjusting measures according to local conditions, reflecting that the Hakka people's life attitude is fitting in with the nature.
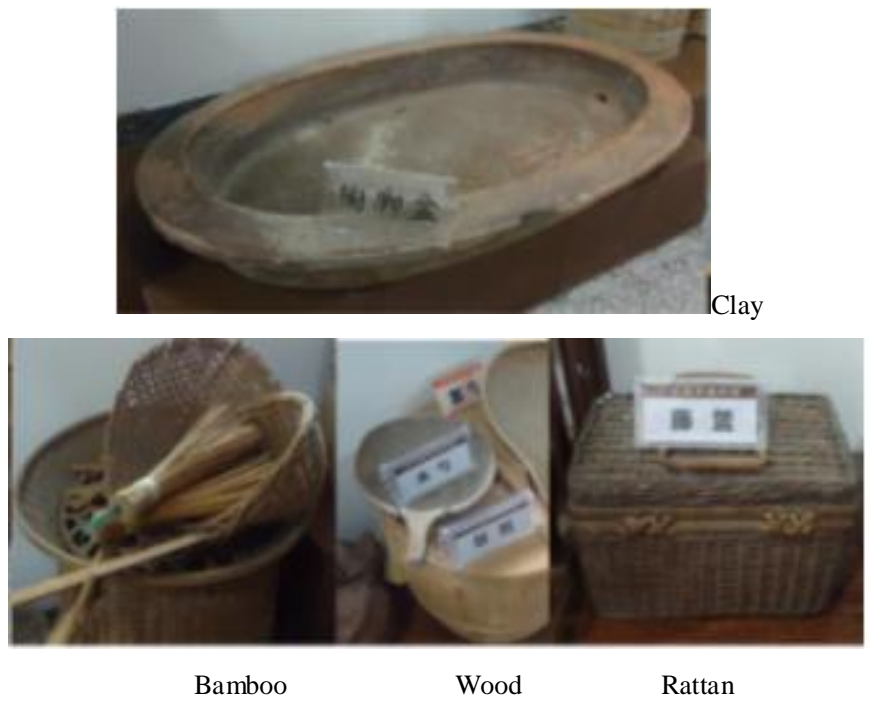

Fig. 1. Native creation material (taken in Tingchow Museum)

The material selection of Hakka traditional utensils fully fits the current so-called green design and eco-sustainable design concepts, and the use of recyclable materials, low pollution, recyclable, biodegradable have been fully reflected in Hakka traditional utensils. For example, the Hakka people make various kinds of daily necessities using bamboo, including the mat used for threshing, basket for screening, mat for drying grain, bamboo basket for storing goods, fine sieve for food processing, dustpan for daily work, bamboo hat for sun and rain shading and so on [2]. In a sense, selecting bamboo according to local conditions to make utensils is the largest eco-friendly behavior: easy to obtain, easy to transport, easy to handle, easy to degrade, and easy to produce, and it has reduced the impact on environment to the largest extent 
without reducing the performance, which is in line with the requirements of material using in ecological design.

\section{LOW ENERGY CONSUMPTION PRODUCTION TECHNOLOGY}

Production technology is one of the basic conditions for the realization of creation. It relates to the whole process of the formation of utensils, and the final formation of them. One can the following characteristics from Hakka traditional utensils: First, the Hakka production technology maintains a certain stability, which also ensures that the traditional daily utensils maintain the stable shape or structure in a very long period of time; Second, the craftsmen's selection of technology derives from the daily life and production needs, and they create utensils according to different natural conditions and raw materials, having the original characteristics. Specific to the production process, the Hakka people tend to produce utensils that coordinate with nature and society based on natural and social conditions. In production, the Hakka craftsmen often unconsciously choose to reduce the production of links, reduce the waste generated, reduce material consumption, and select the production technology with low consumption. In "Fig. 2", bamboo basket is one of the most common Hakka traditional articles. In the production process, such processes as bamboo selection, bamboo breaking, sieve weaving, ring bowing, combination and finishing are a must, and in the production process, the original characteristics of bamboo are maintained as far as possible, which is full of natural beauty. The ecological features of Hakka in production can be seen from the production of bamboo basket: First, each link of the entire production process is essential, and it cannot become an object even when one link is omitted, thus the production process is reduced to one extreme; Second, the thin bamboo strips are weaved in a crossing way, utilizing the space reasonably, and distance is remained between strips of the bamboo, to reduce the use of material as much as possible while ensuring the usability and reliability; Third, in the entire production process, only bamboo is used. Bamboo is satisfying different functional needs through different geometric forms, which has reduced waste as much as possible, and simplifies the material recycling process as well; moreover, the technology of making bamboo has great substitutability, as Hakka people have more than 100 kinds of methods to make bamboo basket. Such ecological features are not only presented in the production of bamboo basket, but they have the same technology selection in stone, wooden and other utensils.

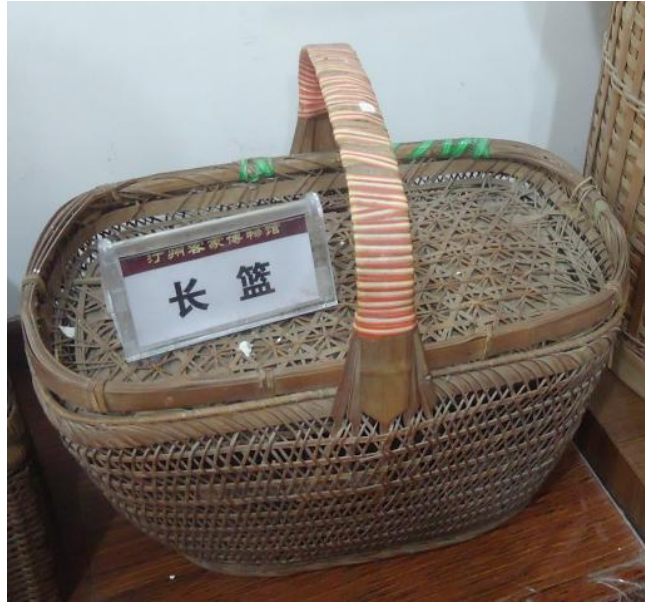

Fig. 2. Long basket (taken in Tingchow Museum)

\section{WAYS TO EXTEND LIFECYCLE}

In view of the extension of product life cycle can improve the efficiency of resource utilization and reduce the negative impact of waste products on the environment, thus the extension of lifecycle of products is one of the core of ecodesign. Contemporary eco-design advocates achieve recycling and reuse of product materials and components through such design principles, methods and tools as durability, remanufacturing, reuse, easy disassembling, recycling, and safe disposal. There is no such written principle in Hakka creation traditions, but in the specific creation and using of utensils, these principles are obviously manifested.

In the Hakka traditional utensils, the extension of product lifecycle and the improvement of product use efficiency are obvious, mainly embodied in: First, most of the utensils are durable type, and the position easy to damage is provided with strengthen treatment. As mentioned above, the long basket, the bottom and mouth are the main force-carrying positions, so in the production process, special consideration are given, and the strength of bamboo ring is significantly higher than other parts. Second, the damaged parts are usually easy to repair. For example, the bamboo, rattan and the like products are usually made with interpenetration structure [4], if there is damage, you can only repair the damaged products, which not only reduces the cost of use, but also reduces material consumption. Third, the core part of many utensils can be disassembled nondestructively. As for the long basket, the big circle at the mouth, the small circle at the bottom and the handle are easy to be removed from the damaged utensils for reuse, on the one hand, it can reduce the "bow circle" link in production, on the other hand, it can also reduce the use of materials. Fourth, product reprocessing, repeated use. For example, the Hakka people will deepen the grinding groove through "washing mill" when the mill surface is worn to a certain degree in the use of stone mill, thus the mill become thin slowly, but its function is always maintained very well. Fifth, upon the utensil is completely scrapped, the material can also be used as fuel for life. The raw materials have played a role from selection to burning. There are various methods and ways to extend 
lifecycle of Hakka traditional daily utensils, such unconscious eco-design approach is worth learning and reference.

\section{CREATION CONCEPT OF PRODUCING ARTICLES FOR PRACTICAL USE}

In the Hakka traditional artifacts, the most prominent design concept is "producing articles for practical use", "shaping according to usage", and in the words of modern terminology, it is the "function of following forms". The traditional daily utensils are mostly made to meet the Hakka people's life and production, with practical use superior to decoration and function superior to aesthetic. According to Adolph Luce, "decoration cannot add any fun", "the pursuit of decoration is essentially a crime against the national economy, eventually leading to the destruction of manpower, money and resources and waste," therefore, the decoration on objects which need not to be decorated means a waste of labor and materials. "[5] In Hakka traditional daily utensils, the Hakka people have almost the same attitude for decoration with Luce. Simple shape, minimal material handling, try to keep the original appearance of the material, make the utensils maintain sufficient economy, reduce the waste of human, money and materials, and correspondingly, it has also reduced impact on environment. [6] In the design of long basket, it is enlarged gradually from the bottom with the top open, the middle space is for storing materials; there is a certain distance between handle and the position of basket to store things, and the handle is in the middle of basket, easy to keep balance; plates can be placed on the upper part of basket, on the one hand, it can cover items in the basket, on the other hand, it can classify the items stored in it; In the process of weaving, thin bamboo strips are given with cross treatment, with hollow positions reserved, which has saved materials, and at the same time, changes in shapes are added; the whole object has no extra decorative parts that do not meet the functional structure. All components have a clear function, even if wrapping a strip of cloth on the handle is for increasing comfort of the handle.

The most prominent Hakka traditional creation concept of "practical use" is "multi-purpose" [7], namely to achieve multiple purpose through one object. From the perspective of resource input and function output, more functional output are obtained with input of the same resource, and the efficiency of using raw materials and manpower is improved. One item has different functions on different occasions, which can be reflected in many Hakka utensils, for example, the long basket can store items (with function similar to the cabinet) and fixed at somewhere, but also can be used as a transport tool to move articles; stone mill can be polish powder and slurry [8]. Similar cases are numerous. From the perspective of embryology, the creator does not always give multifunction to the utensils at the beginning of the creation, but in the process of use, the user uses the utensils from different aspects naturally through conjunction of the physical and chemical forms and geometries of the objects [9]. In this sense, the designer is diverse. This depends on the physical, chemical, and morphological properties of the object itself, and more on the creative use of the user. It also inspires us that the provision of eco-friendly design lies in the designer's design pursuit, but also in the interactive relationship between object and the user. We should go beyond the artifact shape and function itself, to look at the sustainability of objects from a broader perspective of the relationship between objects and people, and the environment. [10]

\section{CONCLUSION}

The Hakka people always maintain a balance and coordination in the reasonable development and utilization of natural resources in the creation activities, which contains a profound ecological design concept, including at least four aspects: First, the localized and renewable selection of natural materials, eco-friendly, fit the natural environment, climatic conditions, easy to regenerate, with low energy consumption and small impact on the environment; Second, in the production process, apply technologies according to materials and needs facing the need for life, compress production links, reduce the use of materials, optimize production technology, which has reduced impact on environment by production process without affecting use; Third, in the process of use, the utensils with good reliability and durability are selected. Timely repair, parts recycling, extend the lifecycle of utensils as much as possible, improve the efficiency of the utilization of materials, and most of the scrapped materials can be used for other purposes, which can reduce the ecological destruction brought by scrapped utensils; Fourth, in terms of the concept of creation, producing articles for practical use, set shapes according to purpose, less decoration, highlight the usability of materials, saving materials, human and financial resources, and all these fit the viewpoint of resource saving of current ecological design. The natural ecological respect presented in Hakka traditional creation activities is amazing. The designer, apart from excitement, need to dig in-depth the concept in it, so that it can obtain new life in the contemporary production conditions, natural conditions, and consumption environment.

\section{REFERENCES}

[1] Victor Papanek,Zhou Bo,Zhao Yan.The Green Imperative:Ecology and Ethics in Design and Architecture[M].China CITIC Press,2013,10:158

[2] Liao Kaishun,Liu Shanqun.Rock hakka narrate theory[M].Henan People's Publishing House, 2012,10:120-134

[3] Tao jing.Since the scheme design phase of product life cycle oriented design method research [D], Shanghai Jiaotong University,Ph.D.Dissertation,2013,12:3

[4] Liu li.Jiangxi hakka traditional culture and bamboo utensils association studies [J].Journal of popular culture:academic, 2014(9):47-48.

[5] The Design History Compilation in Design School of CAFA.Design Gospel:Collection of Western Modern Design[M].Jiangsu Fine Arts Publishing House, 2009,8:185-189

[6] Liang Shengping.People of hakka traditional design research [D],Ph.D. Dissertation, NanJing Art Institute,2010,5

[7] Fan Qinman.Advanced design ideas of Chinese traditional creation [J]. Packaging Engineering, 2008.08:159-162

[8] Qian Tieyu,Meng Yonggang. Harmonious viewpoint of theory of traditional Chinese creation thought enlightenment to modern product design [J]. Packaging Engineering, 2006.05:276-294

[9] TU Delft., WeiYu Ni.DELFT Design Guide[M].Huazhong University of Science and Technology Press, 2015, 10: 20-21

[10] Shen Weiguo.Product design under the influence of the traditional ecological aesthetics research [D],Jiangnan University Professional Master Degree Thesis.2008,8:2 